\title{
Contenido
}

\section{Rector UAA}

Dr. Francisco Javier Avelar González

Decano del Centro de Ciencias de Ia Salud

MCE Jorge Prieto Macías

Editor

Dr. José de la Torre Alcocer

\section{Comité editorial}

Dra. Ma. del Carmen Terrones Saldívar

Dr. Alejandro Rosas Cabral

Dr. Luis Muñoz Fernández

Dr. Efrén Flores Álvarez

MCO Sergio Ramírez González

MCE Víctor Federico Rodríguez Nava

Dr. Juan Manuel Márquez Romero

\section{Consejo editorial}

Centro de Ciencias de la Salud. UAA

Dr. Antonio Ávila Storer

Dr. Eduardo David Poletti

MSP. Raúl Arias Ulloa

Dr. Jesús Martín Galaviz de Anda

\section{Regional.}

Centro de Ciencias Básicas. UAA

Dr. Rafael Gutiérrez Campos.

Centenario Hospital Miguel Hidalgo.

Dr. Carlos Alberto Domínguez Reyes.

Hospital Cardiológica. Aguascalientes

Dr. Guillermo Llamas Esperón

Instituto de Servicios de la Salud del Estado de Aguascalientes

Dr. Javier Góngora Ortega

\section{Nacional}

Hospital de Oncología CMN Siglo XXI

Dr. Pedro Luna Pérez

Hospital Metropolitano México, D. F.

Dr. Eugenio Vázquez Meraz

Instituto Nacional de Ciencias Médicas y Nu-

trición Salvador Zubirán. México, D. F.

Dr. Oswaldo Mutchinik Baringoltz

Universidad de Guadalajara

Dr. Humberto Arenas Márquez

Universidad Autónoma de San Luis Potosí

Dr. Enrique Torre López

Universidad Nacional Autónoma de México

Dr. José Narro Robles

\section{Internacional}

Universidad de California en Los Ángeles.

Dr. Adrián R. Ortega

\section{Traducción}

MCB Ma del Rocío Muñoz Sandoval

\section{Corrección de estilo}

LLH Paloma del Carmen Prieto Terrones

\section{Distribución}

Lic. Martha Elena Reyes Robles

\section{Diseño y formación editorial}

Lic. Rocío Caso Bulnes

Luxmédica: Órgano oficial del Centro de Ciencias de la Salud de la UAA. Los artículos publicados en esta revista son de entera responsabilidad de los autores.

\section{Editorial}

3-10 Correlación entre el nivel de dependencia funcional del adulto mayor y nivel de sobrecarga del cuidador primario en un hospital público de Aguascalientes

Correlation between the level of functional dependence of the elderly and the level of overload of the primary caregiver in a public hospital of Aguascalientes Martínez-García Itzeen Itzamatul Guadalupe, Gallardo-García David, González-Benitez María Fernanda, Guel-Cruz Karla del Rocío, Aguilera-Ibarra Karen Dinhora, González-Flores Silvia Patricia

| |- 7 Nivel de conocimiento del personal de enfermería en reanimación cardiopulmonar, relacionado con factores laborales en los servicios de urgencias de un hospital

Level of knowledge of cardiopulmonary resuscitation in nursing staff, related to labor factors at the emergency room of a hospital

Cruz-López Juan Pablo, Serrano-Salas Diana Karen, Jiménez-Jiménez Herminia, Mosqueda-López

Adriana, Sánchez-Olivares Claudia, Guerrero-Mojica Nery

19-27 Frecuencia de complicaciones en pacientes con diabetes mellitus tipo 2 de un hospital de segundo nivel en Aguascalientes

Frequency of complications in patients with type 2 diabetes mellitus of a second level hospital in Aguascalientes

Prieto-Macías Jorge, Terrones-Saldívar Ma del Carmen, Sandoval-Valdez Darío Alejandro, HernándezGonzález Flavio, Valdez-Bocanegra Jesús Damián, Reyes-Robles Martha Elena

29-37 Regulación de la respuesta inmune durante la infección por Mycobacterium tuberculosis. Artículo de revisión

Review of the regulation of the immune response during Mycobacterium tuberculosis infection

Sánchez-Villa José Diego, Preciado de Santos Miguel

\section{9-45 Embarazo molar parcial llevado a término. Reporte de un caso}

Full-term delivery of a partial molar pregnancy: case report

López-Navarro Juan Manuel, Rubén-Carletti Diego, Martínez-Martínez Fátima Crystal, Mendoza-Navarro Miguel Ángel, González-Acevedo Julieta, Martell-Palos Marisa, González-Gutiérrez José Alberto, Torres-

Cabral Georgina, Terrones-Saldívar Ma del Carmen

47-5 I Síndrome Coronario Agudo secundario al uso crónico de esteroides andrógenos anabólicos en joven fisicoculturista. Reporte de un caso Acute coronary syndrome secondary to chronic use of anabolic androgenic steroids in a young bodybuilder: case report

Prieto-Macías Jorge, Gutiérrez Martínez Silvia Patricia, Tavera-Álvarez José Manuel , Ponce-Damián Leonardo David, Uribe-Quiroz María Fernanda, Zacarías-Serafio José Luis. Prieto Gutiérrez Mauricio Armando, Sandoval Rodríguez Eufracino

\section{Desde las Aullas}

\section{5-60Asociación entre enfermedad periodontal y la morbimortalidad en pacientes con enfermedad renal crónica en hemodiálisis}

Association between periodontal disease and morbidity and mortality in patients with chronic kidney disease on hemodialysis

Viana-Rojas Jesús Antonio, Gordillo-Moscoso Antonio Augusto, López-Quijano Juan Manuel, Pozos-

Guillén Amaury de Jesús, Cepeda-Bravo Juan Antonio, Rosas-Cabral Alejandro

LUXMEDICA, Año 12, Núm. 35 enero-abril 2017, es una publicación cuatrimestral editada por la Universidad Autónoma de Aguascalientes a través del Centro de Ciencias de la Salud, Ave. Universidad 940, Ciudad Universitaria, C.P. 20131, Tel. (449) 91084 30, correo-e:josedelat@ prodigy.net.mx. Editor responsable: Dr. Jose de la Torre Alcocer. Reserva de Derechos al Uso Exclusivo No. 04-2010-0929 18281 800-102. ISSN 2007-1655. Impreso en el Departamento de Procesos Gráticos de la Dirección General de Infraestructura Universitaria de la Universidad Autónoma de Aguascalientes, Ave. Universidad 940, Ciudad Universitaria, Edificio 107, C.P. 20131, Tel. (449) 9108443. Este numero de terminó de imprimir en abril de 2017 , con un tiraje de 1,000 ejemplares. Las opciones expresadas por los autores no necesariamente reflejan nacional.

Publicación financiada con recursos PFCE 2016

(). (1)

Esta revista está aceptada en la base de datos bibliográfica de revistas de ciencia y tecnología Periódica. http://periodica.unam.mx

Los trabajos deben dirigirse a: LUX MÉDICA

Consejo Editorial. Centro de Ciencias de la Salud, Universidad Autónoma de Aguascalientes, edificio 107, Campus Universitario Avenida Universidad 940. C.P. 20131, Aguascalientes, Ags., México. Tel.: 01(449) 910 8443. Correo electrónico: josedelat@prodigy.net.mx mcterron@correo.uaa.mx 